\title{
XII.
}

\section{Zwei Fälle von primärem Scheidensarcom bei kleinen Kindern.}

(Aus der Königl. Chirurgischen Klinils zu Halle a. S.)

Von Dr. med. Conrad Frick, prakt. Arzt.

(Hierzu Taf. IX.Fig. 1-3.)

Während es schon lange bekannt ist, dass Neubildungen aller Art (Carcinome, Fibrome, Polypen, Lipome u. s. w.) in allerdings seltenen Fällen von der Scheide ihren Ursprung nehmen können ${ }^{1}$ ), so gehört merkwürdiger Weise die Kenntniss einer zwar auch recht seltenen, aber wie es scheint, in ihrer äusseren Erscheinungsform, wie in ihrem Verlaufe ganz typischen Geschwulstform erst der neueren Zeit an, nehmlich der papillären oder traubigen, sehr bösartigen Scheidensarcome, welche bei Kindern, meist im Alter von 2-3 Jahren zur Beobachtung kommen. Derartige Fälle sind in den letzten Jahren im Ganzen sieben veröffentlicht worden, nehmlich von Sänger ${ }^{2}$ ), Soltmann $n^{3}$, Hauser ${ }^{4}$, Ahlfeld ${ }^{5}$ ), Demme ${ }^{6}$ ), Steinthal ${ }^{7}$, Schustler ${ }^{8}$ ); alle diese Fälle verliefen tödtlich, mit Ausnahme des letztgenannten, dessen Ausgang noch abzuwarten ist. Von einem weiteren Falle von $\mathrm{Babes}^{9}$ ), der vielleicht ebenfalls hierher gehört, ist nur der mikroskopische Befund berichtet. Endlich

1) S. bierüber: Breisky, Die Krankheiten der Vagina. 1886.

2) Arcbiv für Gynäkologie. 1880. XVI. 58.

3) Jahrbuch für Kinderheilkunde. Bd. XVI. S. 418 .

4) Dieses Archiv Bd. 28. 1882.

5) Archiv für Gynäkologie. Bd. XVI. H.'’.

6) XIX. rned. Bericht über die Thätigkeit des Jenner'schen Kinderspitals in Bern 1882. - Graenicher, Ueber einen Fall von congenitalem Sarcom der Vagina. Inaug.-Diss. München 1888.

7) Dieses Arcbiv Bd. 3. 1888.

8) Wiener klin. Wochenschr. 188s. No. 6.

9) v. Ziemssen's Handb. der spec. Path, u. Therap. Bd. XIV. II. S. 475. 
Virchorn's Archio. Bd. CXVII
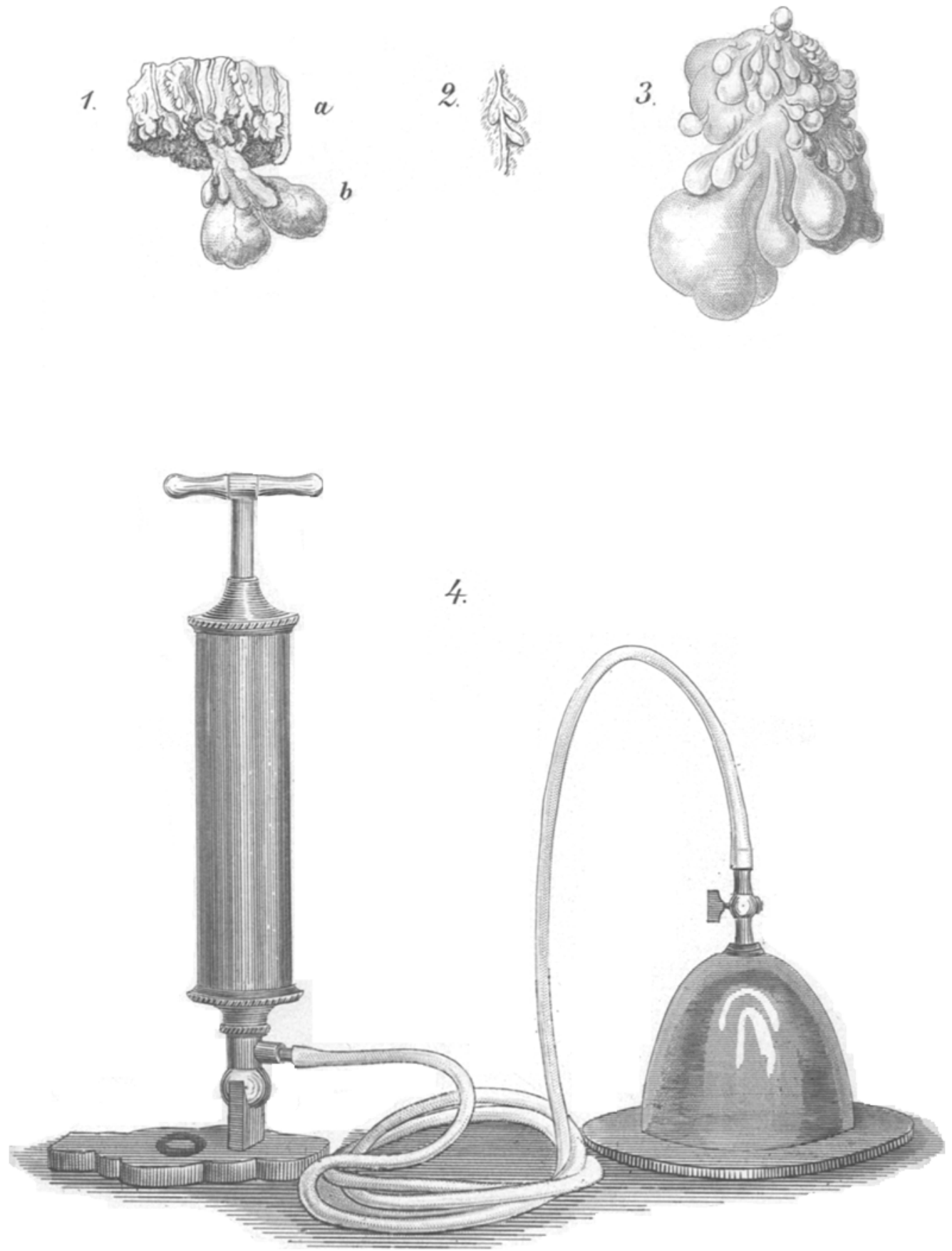

Sichucharidt et Schente del. 
liegen noch von Marsh'), Billroth ${ }^{2}$ ), Guersant ${ }^{3}$ ), Traetzl ${ }^{4}$ ), Wilson ${ }^{5}$ ), Martin ${ }^{6}$ ) Beobachtungen von Scheidengeschwülsten kleiner Kinder vor, die zwar wesentliche Unterschiede gegenüber den von den oben genannten Autoren beschriebenen bösartigen Scheidensarcomen zeigen, deren Vergleichung mit letzterer Goschwulstform aber von grossem Interesse ist.

Den bis jetzt bekannten Fällen von primärem Scheidensarcom kleiner Kinder kann ich noch zwei weitere anreihen, von denen der eine, wie diese, rasch tödtlich verlief, während beim zweiten zwar nach der ersten Operation ein Recidiv eintrat, dann aber, nachdem dasselbe durch eine $z$ weite Operation sehr gründlich entfernt worden war, dauernde Heilung (bis jetzt $2 \frac{3}{4}$ Jahre anhaltend) erreicht wurde.

I. Fall. Polypöses Sarcom der rechten Scheidenwand. Exstirpation. Rasches Recidiv. Tod ${ }^{7}$.

Marie L., 7 Monate alt, wurde durch ibre Eltern im Anfang Februar 1886 zu ihrem Arzt gebracht. Das Kind sah ganz gesund und blihhend aus; in seiner Familie waren Tumoren, soweit bekannt, noch nicht vorgekommen. Wenn das Kind ging, sah man aus der Vulva desselben einen nahezu basel: nussgrossen, ziemlich glatten, blassroth gefärbten Tumor herauskommen; derselbe verursachte weder Sebmerzen noch Blutung oder Ausfluss. Von Anfang Februar bis zum 27. März wuchs der Tumor schnell, die Oberfläche nahm einen mehr polypösen Charakter an. In den letzten Tagen vor der Operation ist der Tumor sehr stark angeschwollen und dunkelgefărbt; er verursacht Urin- und Defäcationsbeschwerden.

Am 27. März 1886 wurde zur Operation geschritten: Das Allgemeinbefinden ist sehr gut; Labia majora und minora von normalem Aussehen;

1) Patholog. Transact. London 1874. S. 198.

2) Billroth, Chirurg. Klinik. Wien $1871-1876$. S. 360 .

3) Journal für Kinderkrankheiten. Bd. XIV. S. 148.

4) Allg. Wien. Med. Ztg. VIII. 1863. No. 30.

5) Med. Times and Gazette. 1876, April. 360 .

6) Zeitschrift für Geburtshülfe und Gynäkologie. 1878. Bd. III. 406.

7) Ich verdanke diese Notizen der Freundlichkeit des Herrn Dr. Roosenburg im $\mathrm{Haag}$, welcher bei seiner Anwesenheit in Halle der Operation des gleich zu beschreibenden Falles II beiwohnte und später die Güte hatte, die Krankengeschichte und das Spirituspräparat des von ihm beobachteten und operirten Kranken an Hrn. Privatdocenten Dr. Schuchardt zu senden. Letzterer machte auf dem in Halle a. S. im Mai 1888 stattfindenden Congress der D. Gesellschaft für Gynäkologie von den beiden Fällen Mittbeilung und stellt das geheilte Kind Kietze vor. 
aus der Vulva, ragt ein dunkelrother, leicht blutender Tumor von keulenförmigem Aussehen heraus, welcher dicht oberbalb des rechten Labium minus an die rechte Vaginalwand angebeftet ist. (Ueber die Grösse und Anbeftungsart der primären Geschwulst ist leider nichts angegeben.) - In Chloroformarkose wird der Tumor; soweit möglich, nach oben zu umschnitten und das Uebrige mit dem scharfen Löffel herausbefördert. Die nicht unerbebliche Blutung wird mittelst Umstechung gestillt, die Wandfläche wurde mit Argent. nitric. geätzt und die Scheide nach gründlicher Desinfection durch Jodoformgaze austamponirt. Der Heilungsverlauf war ganz reactionslos.

Zwei Wochen nach der Operation fingen die Labia majora und minora allmählich an anzuschwellen und hier und dort knotige Verdickungen zu zeigen: Einen Monat nacb der Operation hatte diese Schwellung noch mehr zugenommen, und die ganze Scheide war sebr stark ausgedehnt dureh eine Neubildung von ähnlicher Bescbaffenbeit, wie die vor einem Monat entfernte Geschwulst. Der Tumor ging jetzt von beiden Vaginalwänden aus, am meisten von der rechten. - Eine zweite Operation wurde nicht vorgenommen; das Kind starb in Juli desselben Jabres, also 3-4 Monate nach der Operation.

Die in Spiritus aufbewahrte, durch die Operation entfernte Geschwulst wiegt $9 \mathrm{~g}$ und zeigt folgende Verhältnisse: Sie ist $4,2 \mathrm{~cm} \mathrm{lang,} 3,4 \mathrm{~cm}$ breit, $1,9 \mathrm{~cm}$ dick, ungestielt und sitzt auf einem 1 q $\mathrm{cm}$ grossen Stücke der Vaginalwand. Ihr äusseres Aussehen gleicht einer dicht von Beeren besetzten reifenden Weintraube. Der Tumor besteht aus einer sehr grossen Anzahl kleinerer (stecknadelkopf- bis linsengrosser) und einigen grösseren (erbsen- bis weinbeerengrassen) Polypen, welche alle auf Stielen von wechselnder Länge sitzen und sich nach der Scheidenwand hin $z$ u einer breiteren Fleischmasse vereinigen. Die grösseren Polypen bieten wiederum ein gelapptes Ausseben dar, während die kleineren nur ganz geringe Einkerbungen erkennen lassen.

Der mikroskopische Befund ergab Folgendes: Die Geschwulst, welche von mehrschichtigem Plattenepithel bedeckt ist, besteht aus kleinen und grossen Rundzellen und Spindelzellen mit deutlich sichtbaren Kernen und Kernkörperchen. In der Nähe der Blutgefässe finden sich die Geschwulstzellen meist dichter aneinandergerückt; auch ist an einzelnen Stellen dicht unter dem Epithel die Zellinfiltration etwas stärker, wie an den äbrigen Theilen. - Die Geschwulst ist von zablreichen Blutgefässen durchsetzt, ja stellenweise besteht sie fast ausscbliesslich aus einem Convolut vielfach getheilter und geschlängelter Blutgefässe, zwischen welchen nur ein zartes Maschenwerk feinster Bindegewebsfasern, sowie spärliche in dasselbe verstreute grosse Spindel- und Rundzellen sichtbar sind. Die Gefässe entbalten theils Blut, theils fibrinöse Massen.

Der Tumor muss somit histologisch als ein zum Theil teleangiectatisches Rund- und spindelzellensarcom bezeichnet werden. 
II. Fall. Polypöses Sarcom der hinteren Scheidenwand. Exstirpation. Recidiv nach 8 Monaten. Nocbmalige Operation. Heilung.

Frieda K., $2 \frac{1}{3}$ Jahre alt, blühend und kräftig aussehend, Landmannskind aus Thalschütz bei Lützen, wurde am 17. September 1885 dem Herrn Geh. Med.-Rath von Volkmann zur Untersuchuug vorgeführt. - Das Kind ist früher immer gesund gewesen; die Eltern sind gesund. Im Frübjahr 1885 hatte das Kind den Keuchbusten, welcher 6-8 Wochen andauerte und vielfach heftige Hustenanfälle hervorrief. Seit dieser Zeit, etwa um Pfingsten, hat die Mutter eine Geschwulst, „wie ein paar branne Bohnen“ aus der Scheide hervorragend bemerkt; später nahm die Geschwulst ein mebr weissliches Aussehen an. Während der Erntezeit soll die Geschwulst 4 Wochen bindureb gar nicht zum Vorschein gekommen sein, dann war sie plötzlich wieder da und erwies sich als beträchtlich gewachsen. Unbedeutende Blutungen traten hicr und da auf, einige Male auch Schmerzen, jedoch immer nur ganz unbedeutend. Urin- und Defäeationsbeschwerden waren nicht vorhanden.

Bei der Untersuchung des Kindes in Narkase erwies sich das Hymen intact; im Introitus vaginae fand sich eine haselnussgrosse, polypöse, dunkelrothe Geschwulst, welche mit dünnen, $1-2 \mathrm{~cm}$ langem Stiele der binteren Scheidenwand dicht oberbalb des Scheideneinganges aufsass. Sonstige krankbafte Veränderungen der Beckenorgane sind nicht zu bemerken, die Lymphdrüsen der Weiche sind frei von Anschwellung.

Die von Herrn Geh.-Rath von Volkmann ausgefübrte Operation wurde in Steinschnittlage des Kindes ansgeführt. Die Scheide wurde mit dreizinkigen Wundhaken auseinander gehalten, der polypöse Tumor vorgezogen und mitsammt einem 10 Pfennigstück-grossen Theile der binteren Scheidenwand mit Messer, Scheere und Pincette entfernt. Die Wunde wurde durch einige Catgutnäbte verkleinert und beilte ohne Störung. -- Der von Herrn Dr. Schuchardt aufgenommene anatomische Befund der durch die Operation entfernten Geschwulst ergab Folgendes: "Durch einen gemeinsamen, etwa federkieldicken Stiel befestigt sitzen auf der Scheidenwand zwei polypöse fast $3 \mathrm{~cm}$ lange Gebilde. Sie sind von etwas lappiger Oberfläche, fast haselnussgross, bläulichweiss, vielfach glasig durchscheinend, dem Aussehen und der Consistenz nach den aus ödematösem Bindegewebe bestehenden (Schleim-) Polypen der Nasenhöhlen gleichend, nur vielleicht etwas fester. Die Oberfläche zeigt bier und da kleine Substanzverluste mit Blutungen. Ausser den zwei grösseren Polypen entspringen mehrfach kleine, secundäre Polypen von dem gemeinsamen Stiele, theils als breitaufsitzende, halbkuglige, stecknadelkopf- bis hanfkorngrosse Excrescenzen, theils als etwas grössere, bereits gestielte oder selbst kolbenförmige Gebilde (Fig. 1). Ibr Aussehen unterscheidet sich insofern von dem der grösseren Polypen, als ihnen das glasig durchscheinende Aussehen fehlt, welches so sehr an die Schleimpolypen der Nase erinnert. Sie sind gleichmässig grauröthlich, weich, bier und da mit kleinen punktförmigen Hämorrhagien getüpfelt. Der angręnzende 
Theil der Scheidenwand hat eine beträchtlich derbere Consistenz, als der übrige durchaus weiche Theil der Scheidenschleimhaut. Die Scheidenwand wölbt sich hier zu einer beträcbtlichen Verdickung empor; die Oberfläche der geschwulstartigen Verdickung ist feinhöckrig, ihre Farbe gelblich grau, vielfach röthlich gesprenkelt. In dem oberen Theil des durch die Operation entfernten Stückes der Scheidenwand finden sich fast halblreisförmig angeordnet eine grössere Anzahl kleinster polypöser Auswüchse, theils in der Form halbkugliger Erhebungen, theils wirklich gestielter Anhängsel. Der grosse, aus dem Introitus vaginae vorhängende Polyp ist nur ein besonders ausgebildeter und stark ödematöser derartiger Auswuchs. Von den meisten dieser kleinen Auswüchse lässt sich mit Sicherheit feststellen, dass sie von den zarten Leisten und Falten der Scheidenschleimhant ihren Ursprung genommen haben. Fs entwickeln sich nehmlich aus denseluen anfangs ganz platte, zungenförmige Fortsäłze, welche später grösser, mehr kuglig, endlich gestielt werden" (Fig. 2).

Auch die aus der Scheide herausbängende grosse polypöse Geschwulst ist auf diese Weise entstanden zu denken. Ihre Grösse hat dieselbe ausser ibrem stärkeren Wachsthum auch noch dem in ihr entwickelten, wabrscheinlich durch Druck auf den. Stiel hervorgerufenen Oedem zu danken, Verhältnisse, welche auch für die Entwickelung der Nasenpolypen bekanntlich eine grosse Rolle spielen.

Die mikrosk opische Untersuchung ergab Folgendes: Die Geschwulst zeigt im Allgemeinen einen papillären Bau und ist von mebrfach geschichtetem Plattenepithel bedeckt, welches hesonders an den Stellen, wo die kleineren Polypen vom Haupttumor abgehen, eine dicke Schicht bildet. Man sieht an der Oberfläche des Tumors glatte Zellen, deren Kerne hier und da undeutlicb gefärbt, an einzelnen Stellen aucb gar nicht gefärbt erscheinen; unter diesen eine polymorphe Zellenschicht, welche den Uebergang zur Cylinderzellenschicht bildet. Die Grundsubstanz des Tumors besteht ihrer Zusammensetzung nach meist aus grossen Rundzellen, die besonders unter der Epitheloberfläche dicht gedrängt an einander liegen, aber sich unter einander deutlich und scharf abgrenzen lassen. Die Kerne sind überall deutlich sichtbar; Kernkörperchen sind meist nicht zu erkennen; nur vereinzelt finden sich Kerne mit einem, auch zwei deutlich sichtbaren Kernkörperchen. Zwischen den grossen Rundzellen befinden sich auch eine Anzahl kleinerer Rundzellen und reichliche Spindelzellen. In weiterer Entfernung vom Epithel hört die starke Zellenanhäufung auf und die Zellen finden sich im Vergleich zu der vorher erwähnten massenhaften Zellanhäufung nur noch vereinzelt. Nur in der Umgebung der gleich näher zu erwähnenden Gefässe befinden sich noch starke Infiltrationen von Rundzellen; hier finden sich auch ziemlich viele Spindelzellen, welche moist sich mit ihrer Längsaxe parallel der Gefässwand gestellt haben, und begleiten die Gefässe, hier und da gleichsam wie in Zügen angeordnet. In der Mitte der Geschwulst sind die Zellen im Allgemeinen sehr viel sparsamer und rücken sebr viel weiter auseinander; das zwischenliegende Bindegewebe ist von eigenthümlich heller, 
zarter, faseriger Beschaffenheit; oft findet man nur ein ganz feines Maschenwerk von ausserordentlich dünnen Fäserchen. Wahrscheinlich handelt es sich hierbei um einen Zustand von Oedem in der Geschwulst, welcher durch Compression des Stiels des betreffenden Polypen hervorgerufen ist. Blutgefässe finden sich zahlreich und meist stark gefüllt, namentlich in den mittleren Theilen der Geschwulst.

Sehr lebrreich ist die Untersuchung der Scheidenschleimbaut in unmittelbarer Nähe der Geschwulst. Man sieht hier wie die schon oben erwähnten kleinsten papillären Wucherungen der Schleimhautfalten schon im Grossen und Ganzen denselben Bau darbieten, wie die spätere grosse Geschwulst. Thre Grundsubstanz besteht aus grossen und kleinen Rundzellen und Spindelzellen, ganz wie im Haupttumor, nur vielleicht noch etwas dichter gedrängt. Zahlreiche Blutgefässe, theils stark gefüllt, theils leer, finden sich auch hier; dieselben reichen bis dicht an die Epitheldecke heran und sind gerade unterhalb derselben am stärksten entwickelt. Die bei der Hautgeschwulst beschriebene eigenthümliche Auffaserung des Bindegewebes, welche wabrscheinlich auf einen Zustand von Oedem zuräckzuführen war, find sich bei diesen noch im ersten Stadium ibrer Entwickelung stehenden papillären Wucherungen nicht, weil bei den kleinen Tumoren noch keine Compression des Stiels und dadurch bedingtes Oedem hat eintreten können.

Am 16. October 1885, 4 Wochen nach der Operation, wurde das Kind wieder vorgestellt; die Heilung war eine vollständige. Dagegen mussten die Eitern am 22. Mai des folgenden Jahres wiederum die Hülfe des Herrn Geh. Med.-Rath von Volkmann nachsuchen, denn seit 6 Wochen hatte sich bei dem Kinde, welches inzwischen das Scharlachfieber durchgemacht hatte, wieder eine Geschwulst im Scheidenausgange gezeigt. Dieselbe ist jetzt bedeutend grösser, wie die erste, im Ganzen etwa wallnussgross, wieder von dem eigenthümlicb lappigen, traubigen Bau (Fig. 3), und wird beim Pressen aus der Scheide herausgedrängt. Der. Tumor sitzt mit ziemlich breiter Basis an der hinteren Scheidenwand, an der Stelle der ursprünglichen Geschwulst.

Die Operation wurde wiederum in Narkose und Steinschnittlage vorgenommen. Der Tumor wurde mit einer Muzeux'schen Zange hervorgezogen, die Vagina mit scharfen Haken auseinandergehalten, sodann wurde die ganze untere Hälfte der binteren Scheidenwand mitsammt der Geschwulst bis, in das Septum recto-vaginale hinein mit Messer, Scheere und Pincette exstirpirt. Der Rest der Scheidenwand wurde heruntergezogen und mit drei Nähten an die Haut befestigt; in die entstandene Wundböhle wurde ein Drain eingelegt. - Am 31. Jai ist alles prima intentione geheilt; die Näbte und das Drainrohr können entfernt werden; das Kind wird entlassen.

Das Kind wurde von dem Arzte in seiner Heimath unter Controle behalten; es reigte sich kein Recidiv wieder, vielmehr hat sich das Kind gesund nnd kräftig entwickelt und ist in keiner Beziehung hinter seinen Ge. schwistern zurückgeblieben. Am 26. Mai 1888 wurde das Kind auf dem Congress der Deutschen Gesellschaft für Gynäkologie von Herrn Privat- 


\section{4}

docenten Dr. Schuchardt vorgestellt. Dasselbe sab gesund und blühend ans. Die Untersuchung ergab eine leichte Stenose des Orificinm vaginae, sowie eine von der Operation herrihrende Narbe an der hinteren Commissur. Sonst ergab die äussere Untersuchung nichts Abnormes; der Hymen war infact. Vom Sastdarm aus füblte man die hintere Vaginalwand vollständig weich, ohne Spur einer Neubildung; der Uterus war vollkommen normal gelegen und durcbaus nicht etwa durch den stattgehabten Vernarbungsprozess beruntergezogen ${ }^{1}$ ).

Die mikroskopische Untersuchung der recidiven Gescbwulst ergiebt Folgendes: Der Tumor ist ebenso, wie der vorher beschriebene, von mebrfach geschichtetem Plattenepithel bedeckt. (Man sieht auch bier an der Oberfläche des Tumors platte Zellen, teren Kerne sich scbwach färben. Unter diesen erkennt man wiederum eine Schicht polymorpher Zellen, welche den Uebergang zur Cylinderzellenschicht bildet.) Eine Schicht kleinzelliger Infiltration an der Epithelgrenze, wie sie bei dem ersten Tumor zu finden war, ist hier nicht in derselben Gleichmässigkeit vorhanden; zum Theil findet sich eine solche Infiltration überhaupt nicht vor. Die Grundsubstanz des Tumors besteht aus grossen und kleinen Rundzellen, deren Kerne deutlich gefärbt erscheinen und theilweise ein, auch zwei Kerntörperchen deutlich erkennen lassen. Unter ihnen finden sich eine grosse Anzahl Spindelzellen, welche zum grossen Theil in Zügen und Strängen parallel zur Oberfläche angeordnet sind und an einzelnen Stellen die Hauptmasse der Geschwulst bilden. Starke Zelleninfiltrationen, wie sie sich bei der ersten Geschwulst in der Nähe des Epithels vorfinden, sind hier nur in ganz geringem Maasse vorbanden. Im Allgemeinen entspricht das Bild des Recidivtumors dem der ersten Geschwulst; doch sind die Zellen etwas zablreicher und auch dichter zusammengedrängt, vor Allem aber finden sich zahlreichere Spindelzellen darunter. Das bei der ersten Geschwulst erwähnte eigenthümliche aufgefaserte bindegewebige Stroma findet sich hier nur in geringerem Maasse und keineswegs so charakteristisch ausgeprägt, wie bei der ersten Geschwulst, die Blutgefässe sind ebenfalls reichlich und meist stark gefüllt, einige sind blutleer. Dieselben finden sich sowobl an der Basis und im Centrum des Tumors, besonders aber auch noch unter der Epitheldecke stark entwickelt. Ein so starker Gefässreichthum, wie in dem Falle I, wo die Geschwulst stellenweise einen fast cavernösen Bau batte, ist hier nicht vorhanden.

Da fast bei jedem der oben erwähnten Fälle von Scheidensarcom von den Autoren genaue Analysen der in der Literatur bis dahin bekannt gewordenen Fälle angestellt worden sind, so verzichte ich auf eine Wiedergabe der früheren Beobachtungen und verweise in dieser Beziehung auf meine Dissertation ${ }^{2}$ ). Alle bisher

1) S. Verhandl. d. deutschen Gesellsch. f. Gynäkologie. 1888.

2) C. Frick, Ueber zwei Fälle von primärem Scheidensarcom bei kleinen Kindern. Dissertation, Halle 1888. 
beschriebenen Fälle von Scheidensarcomen kleiner Kinder haben folgende ganz charakteristische, gemeinsame Eigenthümlichkeiten. Das Auffallendste an diesen Geschwülsten ist, dass sie nur bei Kindern im frühesten Lebensalter beobachtet werden und dass sie unter ganz typischer anatomischer Form auftreten. Sie bilden nehmlich traubige, polypöse Geschwülste, theils breitbasig, theils gestielt der Scheidenwand aufsitzend, deren Aussehen am treffendsten mit dem einer Blasenmole verglichen werden kann.

Diese Geschwülste verhalten sich prognostisch offenbar ganz ähnlich, wie die seltenen traubigen Geschwülste des Uterus, welche „im Anfange ihrer Entwickelung auf der Grenze zwischen gut- und bösartigen Neubildungen stehend, in der Regel sehr bald einen sehr bösartigen Charakter annehmen" (Winkel, Lehrbuch der Frauenkraukheiten. Leipzig 1886. S. 430) ${ }^{1}$ ). In der That sind die bisher beschriebenen Fälle von traubigen Sarcomen des Uterus alle tödtlich verlaufen. Auch die analogen Scheidengeschwülste sind sämmtlich bis auf den von von Volkmann operirten Fall tödtlich verlaufen. Die besonderen Eigenthümlichkeiten des letzteren Falles sollen gleich noch näher gekennzeichnet werden. Die Bösartigkeit der traubigen Scheidensarcome ist zunächst eine rein örtliche; abgesehen von den durch die Grösse der Geschwulst bedingten Störungen treten nach Exstirpation rasche Recidive auf und die Geschwulst hat grosse Neigung sich in der Nachbarschaft zu verbreiten, theils durch continuirlicho Wucherung, theils in Form von neuen an bisher noch nicht erkrankten Theilen der Scheidenwand entstehenden Geschwülsten.

Von der Scheide aus greift die Geschwulst im weiteren Verlauf auf die Harnblase (beim Sitz der primären Geschwulst an der vorderen Vaginalwand unter Umständen sehr frübzeitig Soltmann) über, ferner auf die Gebärmutter, die Tuben, Ovarien und Ligamenta lata. Durch Ulceration und Perforation eines Sarcomknotens kann es zu tödtlicher Peritonitis kommen. (Sänger). - Schwellung und sarcomatöse Entartung der Lymph-

1) Vgl. die neueste Arbeit bierüber von Pernice "Ueber ein traubjges Myosarcoma striocellulare uteri", dieses Archiv. 1888. Bd. 113. 
drïsen sind nur in drei Fällen (Sänger, Ahlfeld, Demme) beschrieben. - Von sonstigen Metastasen ist nur in einem Falle etwas berichtet (Demme). Hier wurden im letzten Stadium der Erkrankung zunächst die benachbarten Lymphdrüsen in Mitleidenschaft gezogen, so namentlich die auf der rechten Seite gelegenen; die Vergrösserung der Inguinaldrüsen war intra vitam nachweisbar, bei der Section zeigten sich auch die Retroperitonäal- und Iliacaldrüsen ansehnlich sarcomatös infiltrirt. Späterhin wurde auch das linke Ovarium in Mitleidenschaft gezogen und in einen sarcomatösen Tumor umgewandelt.

Die Dauer des Kranheitsverlaufes ist verschieden; im Falle Roosenburg trat der Tod 6 Monate nach dem Bemerkbarwerden der Geschwulst ein; im Steinthal'schen Falle dagegen erst nach 20 Monaten. Im Mittel führte die Erkrankung binnen 12 Monaten zum Tode. - Im Falle Babes trat allerdings erst nach drei Jahren ein Recidiv ein. Der Fall Demme ist insofern ganz ungewöhnlich, als die Geschwulst $5 \frac{1}{2}$ Jahre bestanden hatte, ohne nennenswerthe Symptome, abgesehen von kleinen Blutungen und vorübergehenden Schmerzen beim Wasserlassen; dann erst traten Beschwerden auf und wiederholte Recidive führten binnen Jahresfrist zum Tode.

Das Auftreten der Recidive schwankt zwischen 6 Tagen (Hauser) und 8 Monaten (Fall II) nach der Excision; meist entwickelt sich das Recidiv an der alten Stelle, doch kann auch von einem ganz anderen Theile der Scheide und unabhängig von dem primären Tumor eine zweite Geschwulst entstehen, so in dem Demme'schen Falle, wo das zweite Recidiv auf der linken Vaginalwand sass, während der Haupttumor und das erste Recidiv von der rechten Vaginalwand ausging. Nach Entfernung dieses Recidivs blieb die Vagina gesund; doch entwickelte sich bald eine Recidivgeschwulst an der hinteren Blasenwand, sowie Metastasen in den Ovarien und Lymphdrüsen, welche den Tod bald herbeiführten. Aehnlich verhält es sich im Fall I, wo die 14 Tage nach der Operation auftretende Recidivgeschwulst von beiden Vaginalwänden ausging, während der primäre Tumor seinen Sitz auf der rechten Scheidenwand gehabt hatte. 
Was die Insertionsstelle der primären Geschwulst anbetrifft, so befand sie sich in den zuerst erwähnten neun Fällen fünfmal an der vorderen (Sänger, Soltmann, Ahlfeld, Hauser, Schustler), dreimal an der seitlichen (Fall I, Demme, Steinthal) and einmal an der hinteren Wand der Scheide (Fall II). Die Geschwülste sind theils gestielt, theils sitzen sie breitbasig mit pfennigstück- bis thalergrosser Basis der Vaginalwand auf.

Die Symptome der Geschwulst bestehen zunächst in Schmerzen (besonders beim Urinlassen), Blutungen, eitrigem Ausfluss aus der Scheide. Bei stark wachsendem Tumor stellen sich in Folge der durch die Beckenenge des Kindes bedingten Raumbeschränkung bald heftigere Beschwerden ein; Tenesmus, Incontinentia urinae, Retentio urinae, Cystitis (ev. Nephritis, Pyelonephritis), Peritonitis; auch Loslösung einzelner Geschwulsttheile ist beobachtet worden (Sänger). Bei sehr grossen Geschwülsten kann es zu Hervorwölbungen des Hypogastriums, zu Circulations- und nervösen Störungen durch Druck der Geschwulst auf Gefäss- und Nervenstämme kommen.

Wohl ist zu unterscheiden bezüglich der Symptome, ob die Geschwulst sich innerhalb der Vagina weiter entwickelt, oder ob sie bald nach aussen tritt; im letzteren Falle kann das Allgemeinbefinden längere Zeit relativ ungestört bleiben. $\mathrm{Za}$ den schwereren und Gefahr bringenden Symptomen, welche bei den intravaginal sich entwickelnden Geschwülsten (in Folge der Beckenenge beim Kinde) durch Druck auf Urethra, Blase und Mastdarm sehr bald hervorgerufen werden, kommt es in solchen Fällen erst später.

Die operative Behandlung der polypösen Scheidensarcome kleiner Kinder hatte bisher keine erfreulichen Ergebnisse zu verzeichnen, so dass sie fast als aussichtslos betrachtet werden musste. Freilich war ein Theil der beschriebenen Fälle auch in einem Stadium zur Behandlung gekommen, wo an eine radicale Entfernung der Geschwulst nicht mehr zu denken war; so in dem Sänger'schen Falle, der sicher überhaupt nicht operirt worden wäre, wenn man damals schon die ausserordentliche Bösartigkeit derartiger Geschwülste gekannt hätte. In anderen Fällen, z. B. Hauser, Steinthal, ist die erste Operation wahrscheinlich nicht 


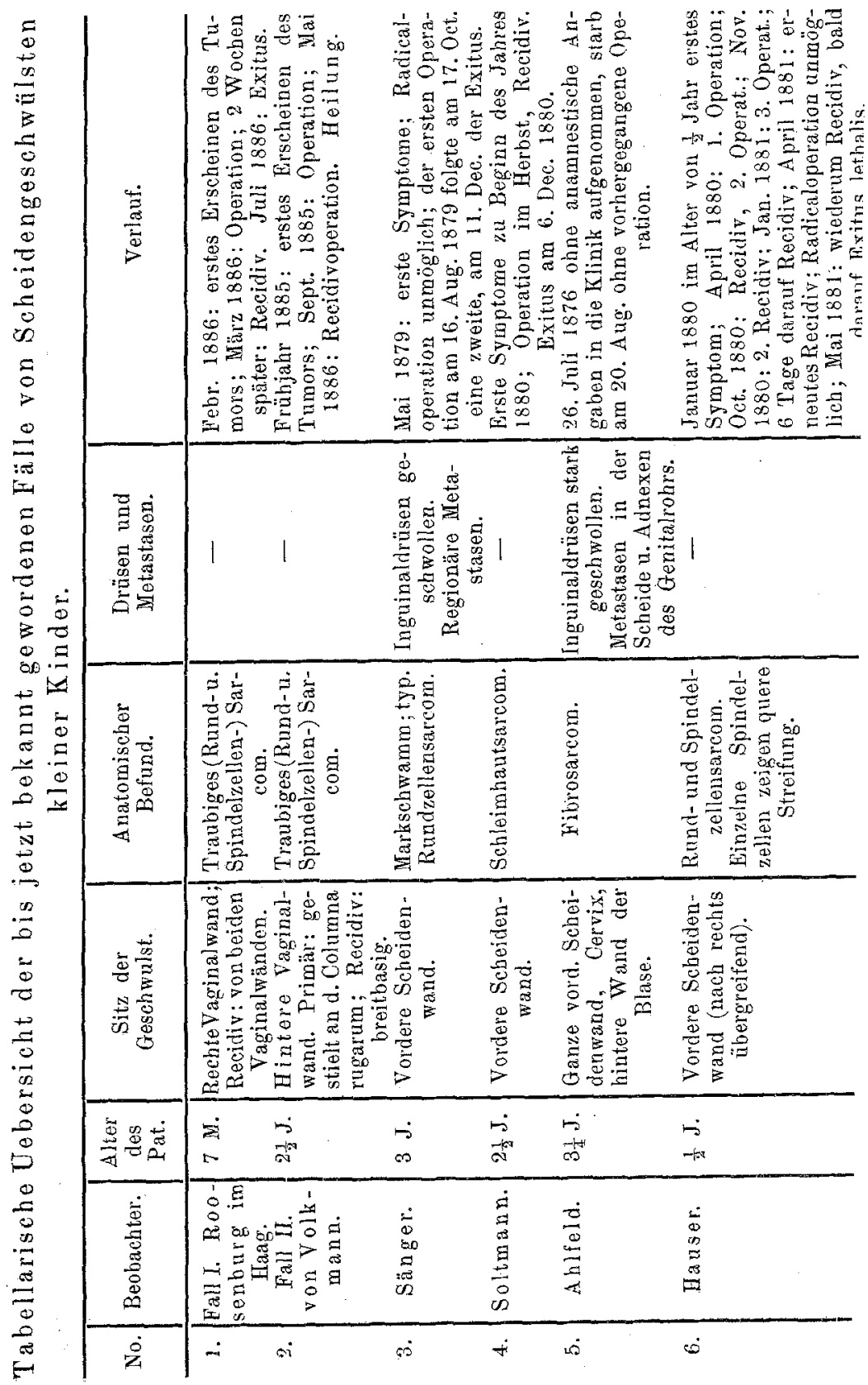




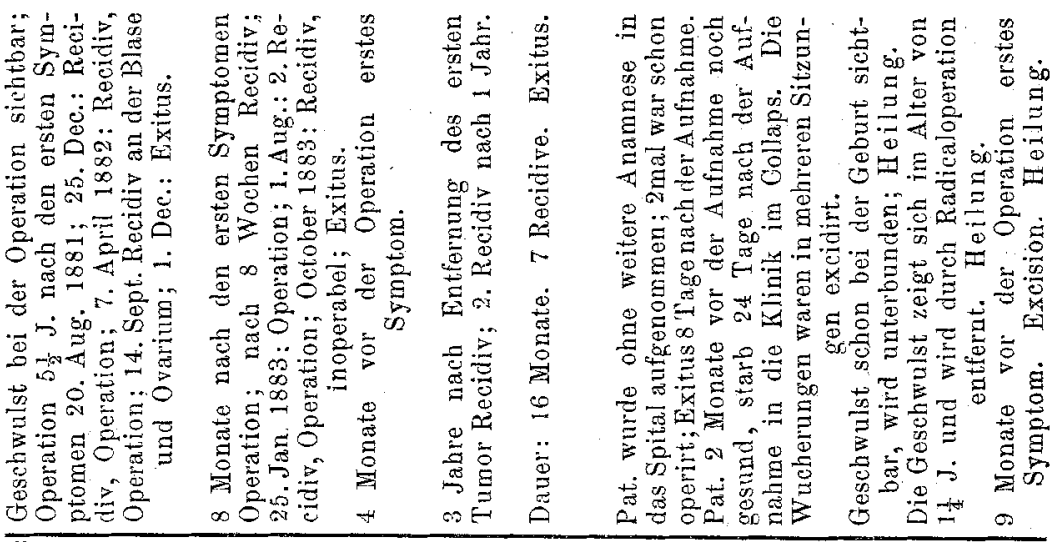

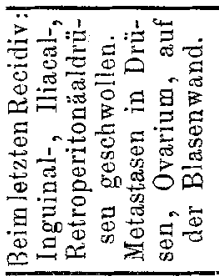

\begin{tabular}{|c|c|c|c|c|c|c|c|c|c|}
\hline 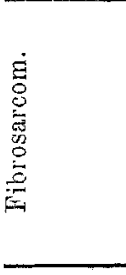 & 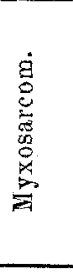 & 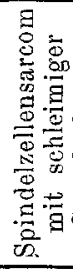 & 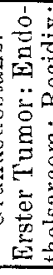 & 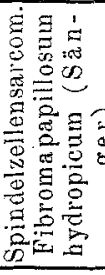 & 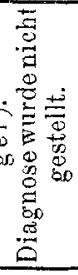 & 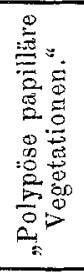 & 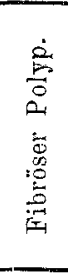 & 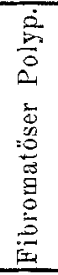 & 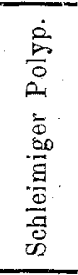 \\
\hline 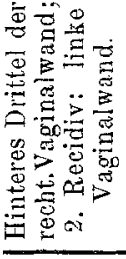 & 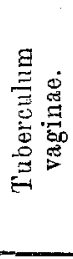 & 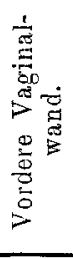 & $\mid$ & 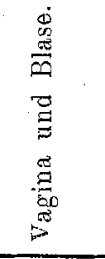 & 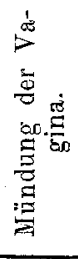 & 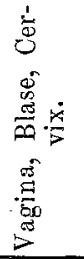 & \multicolumn{3}{|c|}{ 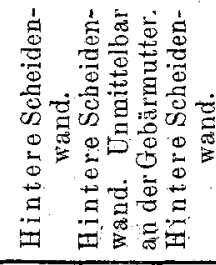 } \\
\hline 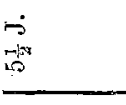 & $\stackrel{\circ}{\infty}$ & $\dot{+}$ & $\stackrel{3}{\infty}$ & $\vec{m}$ & $\underset{\infty \rightarrow+\infty}{\infty}$ & 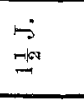 & $\begin{array}{l}\vec{D} \\
\dot{D} \\
\ddot{\omega} \\
\end{array}$ & $\underset{j}{-i}$ & $\dot{m}_{-\infty}^{-\infty}$ \\
\hline $\begin{array}{l}\stackrel{0}{\Xi} \\
\stackrel{g}{a} \\
\stackrel{g}{0} \\
\stackrel{0}{0}\end{array}$ & 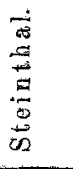 & 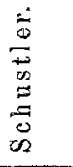 & $\begin{array}{l}\dot{1} \\
0 \\
0 \\
0 \\
\infty \\
0\end{array}$ & $\begin{array}{l}\dot{\Delta} \\
\frac{\infty}{2} \\
\stackrel{5}{3}\end{array}$ & 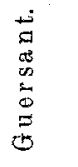 & 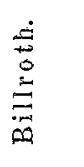 & $\begin{array}{l}\stackrel{\Xi}{\Xi} \\
\stackrel{\Xi}{ \pm} \\
\stackrel{\Xi}{\Xi}\end{array}$ & 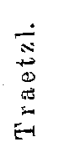 & 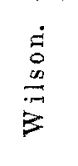 \\
\hline$\therefore$ & $\infty$ & $\dot{0}$ & $\dot{\theta}$ & $\vec{m}$ & $\underset{\sim}{\sigma}$ & $\stackrel{\infty}{7}$ & $\stackrel{\stackrel{+}{-}}{17^{*}}$ & $\stackrel{\leftrightarrow}{\sim}$ & $\dot{\Theta}$ \\
\hline
\end{tabular}


mit der Gründlichkeit vorgenommen worden, wie wir sie nach unseren jetzigen Erfahrungen gerade für diese Geschwülste verlangen müssen. - Der Soltmann'sche Fall stellt ein verhältnissmässig frühes Stadium vor, welches aber durch die unglückliche Complication mit sehr frühzeitigem Uebergreifen auf die Blase durch Urämie sehr bald zum Tode führte. - Im Falle Ahlfeld konnte wegen der colossalen Ausbreitung der Geschwulst im ganzen kleinen Becken überhaupt an eine Operation nicht gedacht werden; das Kind ging kurz, nachdem es in die Klinik gebracht worden war, an Cachexie zu Grunde. - Im Fall Demme wurde zuerst anscheinend mit Erfolg operirt; bei dem letzten Recidiv allerdings hatte der Krankheitsprozess schon eine solche Ausdehnung angenommen, dass eine Operation aussichtslos erscheinen musste.

In unserem Fall I (Dr. Roosenburg) hatte sich 14 Tage nach der Excision der primären Geschwulst schon wieder eine weit grössere Recidivgeschwulst entwickelt; von einer zweiten Operation wurde Abstand genommen.

Der von von Volkmann operirte Fall II lehrt uns aber glücklicher Weise, dass durch eine möglichst frühzeitige radicale Operation ein günstiger Erfolg zu erzielen ist. Allerdings muss ja zugegeben werden, dass auch in diesem anscheinend geheilten Falle im Laufe der Jahre immer noch einmal ein Recidiv auftreten kann. Bis jetzt ist, abgesehen vom Schustler'schen Falle, dessen Verlauf erst noch abzuwarten ist ${ }^{1}$ ), der Fall II der einzige, bei dem ein günstiges Resultat erzielt worden ist. Besonders bemerkenswerth ist bei diesem Falle, dass er sich von allen bisher beschriebenen wesentlich durch die Insertionsstelle der Geschwulst unterschied, welche einzig in diesem Falle an der hinteren Vaginalwand gelegen war. - An und für sich werden die an der hinteren Scheidenwand sitzenden Geschwülste eine günstigere Prognose zulassen, als die übrigen, da ein Uebergreifen der Geschwulst auf das Rectum verhältnissmässig weniger gefährlich ist, als die Ausbreitung nach der Blase. Vielleicht ist aber auch der bisher

1) Derselbe ist inzwischen auch tödtlich verlaufen. $\mathrm{S}$. Wiener klin. Wochenschr. 1889. No.6-11. (Kolisko.) 
so günstige Verlauf des Falles II mit dadurch bedingt, dass derselbe eben durch seinen Sitz in der hinteren Scheidenwand sich mehr den gutartigen Formen von Geschwülsten nähert, wie sie Martin, Traetzl, Wilson beschrieben haben. Von diesen Fällen sind die beiden ersten als fibröse Polypen, der letzte als schleimiger Polyp beschrieben worden. In jedem Falle wurde der Tumor an der Basis unterbunden; schon nach wenigen Tagen fiel die Ligatur $a b$ und völlige Heilung trat ein. Auch in diesen Fällen sass der Tumor auf der hinteren Scheidenwand und in allen drei Fällen wurde ein günstiger Erfolg erzielt. Indess muss hervorgehoben werden, dass der makroskopische und mikroskopische Bau der von von Volkmann operirten Geschwulst durchaus mit den bösartig verlaufenden polypösen Scheidensarcomen der vorderen Vaginalwand übereinstimmt und sich also von den oben genannten gutartigen Geschwülsten histologisch sehr wesentlich unterscheidet.

Für die operative Behandlung ergiebt sich nach den oben mitgetheilten Befunden der Grundsatz nicht nur den Tumor mit Stiel und Basis zu entfernen, sondern vor Allem auch möglichst viel von der Vaginalwand aus der Umgebung der Geschwulst mit fortzunehmen, da auch die umgebende Vaginalwand theilweise schon papillomatöse Wucherungen trägt, die als Vorstadien der sarcomatösen Tumoren aufzufassen sind.

\section{Erklärung der Abbildungen.}

Taf. IX. Fig. 1-3.

Polypöses Sarcom der hinteren Vaginalwand von Frieda Kietze, $2 \frac{1}{2}$ Jahre alt.

Fig. 1. Die durch die erste Operation entfernte Gescbwulst. a Entferntes Stïck der Scheidenwand (Wucherungen der Schleimhantfalten). b Vor der Vulva liegende Gescbwulst.

Fig. 2. Eine Schleimhautfalte mit kleinsten blattfömigen Wucherungen bei Loupenvergrösserung.

Fig. 3. Recidive Geschwulst, 8 Monate später entfernt. 